> Les criblages phénotypiques visent à identifier des molécules capables d'interférer avec une fonction cellulaire d'intérêt à partir de collections de molécules chimiques directement testées sur les cellules en culture. C'est une stratégie risquée, mais puissante, pour découvrir à la fois de nouvelles cibles thérapeutiques et leurs régulateurs pharmacologiques. Forts d'une expérience de près de 10 ans dans ce domaine, nous soulignons dans cet article les avantages et les difficultés inhérents à ce type d'approche, et présentons des solutions pour surmonter plusieurs types d'obstacles. Une communauté scientifique nationale s'est structurée autour de diverses approches de criblage que nous présentons également. <

«Il n'y a rien de vivant dans une cellule sauf l'ensemble. »

Perturber un système biologique est une des approches permettant de le comprendre. Inspiré par l'ère génétique où la fonction des gènes a été déduite de l'étude de collections de mutants générés sur des organismes modèles (drosophile, souris, poisson zèbre, nématodes, plantes, etc.), tout un ensemble de stratégies complémentaires a été développé pour perturber la fonction des gènes ou des protéines, aussi bien dans des organismes que dans des cellules en culture. Les anticorps inhibiteurs, les invalidations ou les surexpressions géniques, et les ARN interférents ont ainsi été largement utilisés pour analyser les fonctions des protéines. Les molécules issues de la chimie représentent des outils puissants et complémentaires des approches génétiques pour étudier les fonctions des gènes et des protéines. Capables d'agir en quelques secondes, d'une façon dépendante de la dose et souvent réversible, elles autorisent un contrôle temporel sur la fonction des protéines afin de sonder les phénomènes dynamiques et de s'affranchir, tout au moins pour les échelles de temps courtes, des mécanismes d'adaptation observés lors d'invalida-

\section{Les criblages \\ phénotypiques \\ ou comment faire \\ d'une pierre \\ deux coups}

Découvrir la cible et la molécule pharmacologique capable de la réguler

Renaud Prudent ${ }^{1}$, Emmanuelle Soleilhac ${ }^{2}$, Caroline Barette $^{2}$, Marie-Odile Fauvarque ${ }^{2}$, Laurence Lafanechère ${ }^{1}$

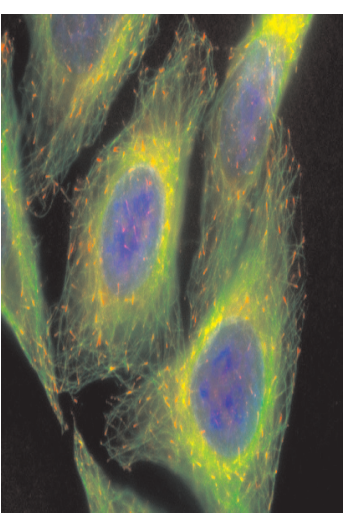

${ }^{1}$ Institut Albert Bonniot, CRI Inserm/Université Joseph Fourier (UJF) U823, équipe 3

Polarité, développement et cancer, rond-point de la Chantourne, 38706 La Tronche Cedex, France ;

${ }^{2}$ Institut de recherches en technologies et sciences pour le vivant (IRTSV), Laboratoire de biologie à grande échelle (LBGE), Gen\&Chem, Centre de criblage de molécules bioactives (CMBA), U1038 Inserm/ CEA/UJF, CEA Grenoble, 17, rue des Martyrs, 38054 Grenoble Cedex 09, France. laurence.lafanechere@

ujf-grenoble.fr de la chimie, la chémobiologie, initiée à la fin des années 1990, est désormais une discipline à part entière en recherche académique. Elle vise à systématiser l'emploi de petites molécules pour l'exploration des fonctions biologiques, à l'instar des mutations en génétique (Figure 1).

Dans les approches dites de « génétique chimique directe », des collections de molécules (chimiothèques) sont criblées sur des cellules (ou plus rarement sur des organismes modèles), à l'aide de robots et de tests adaptés (criblages phénotypiques). Les molécules capables d'induire un phénotype d'intérêt sont ainsi sélectionnées [2]. L'enjeu est ensuite d'identifier la cible intracellulaire des molécules sélectionnées. Dans de nombreux cas, cette étape, bien que techniquement difficile, a été achevée avec succès, contribuant à l'avancée des connaissances fondamentales du vivant [3-5].

En s'adressant à une protéine cible dans son contexte physiologique, les criblages phénotypiques présentent un grand intérêt par rapport aux criblages réalisés sur protéines isolées. Les composés identifiés sont, en effet, d'emblée sélectionnés pour leur capacité à traverser la membrane 


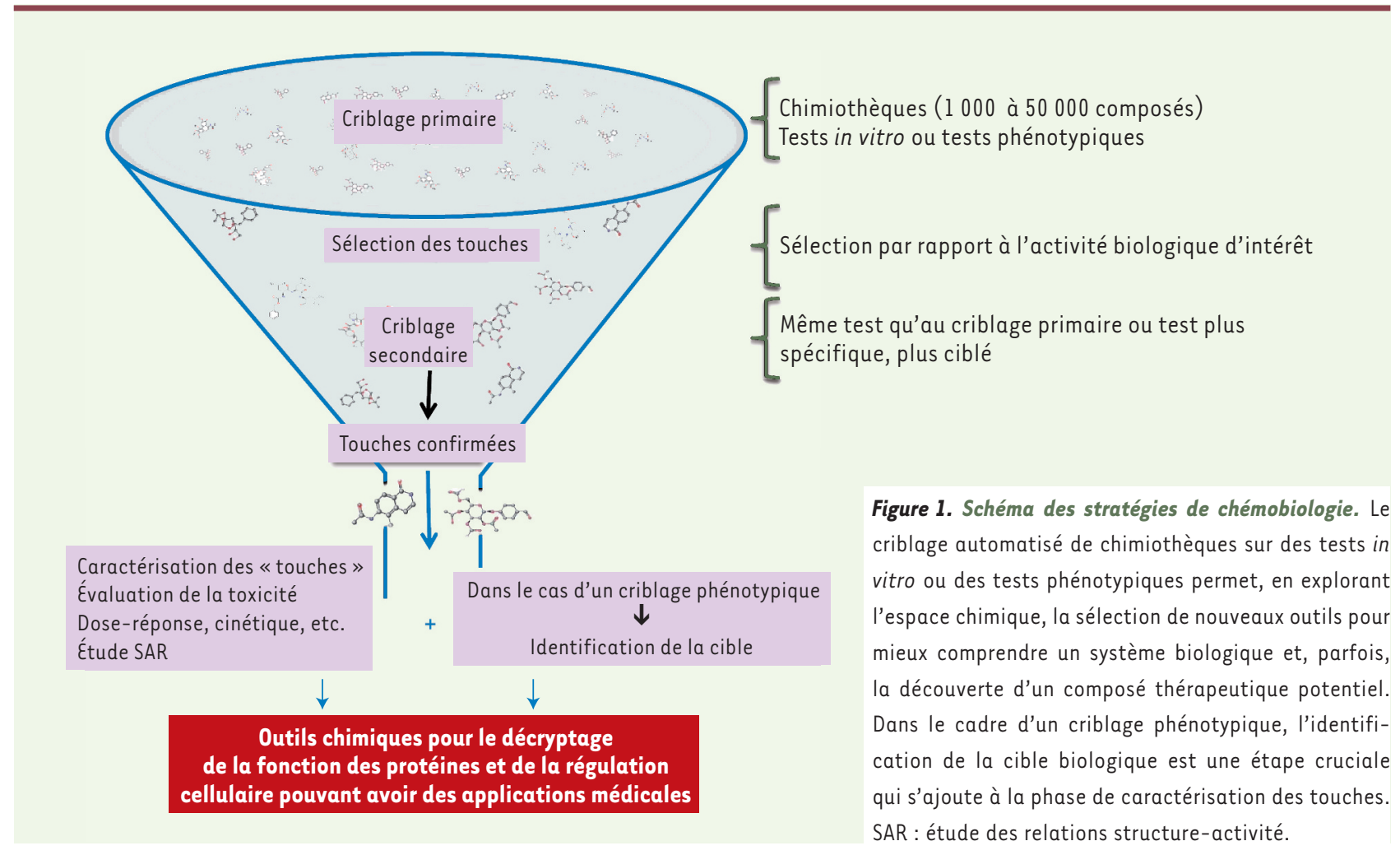

plasmique et pour leur stabilité dans un milieu physiologique. Ces propriétés sont essentielles, aussi bien pour le développement de molécules thérapeutiques, que pour leur utilisation en recherche fondamentale, notamment pour l'étude de phénomènes dynamiques en vidéo microscopie. Surtout, l'atout majeur des criblages sur cellules entières est qu'ils sondent tous les acteurs potentiels, connus ou inconnus du processus cellulaire étudié, pour aboutir à la sélection de la cible la plus pertinente [1]. Cette approche, sans a priori, est génératrice de découvertes inattendues précieuses (nouvelles cibles, nouveau mode d'action) pour la compréhension du vivant et le développement de nouvelles stratégies médicales. Ainsi, c'est principalement grâce à ce type d'approche que de nouvelles entités médicamenteuses, présentant des modes d'action nouveaux, ont été identifiées [6].

Les stratégies de criblages phénotypiques restent néanmoins risquées. Elles imposent une réflexion approfondie sur les tests à mettre en œuvre et les chimiothèques à employer. Elles nécessitent aussi d'anticiper sur la stratégie à adopter pour l'identification ultérieure des cibles. À Grenoble, nous avons mis en place et utilisons depuis plus d'une dizaine d'années une plateforme académique de criblage automatisé, le Centre de criblage pour des molécules bio-actives(CMBA), adaptée à la réalisation de tests cellulaires (Figure 2).

\section{Tests cellulaires pour les criblages phénotypiques}

Par rapport à des recherches guidées par des hypothèses, les criblages phénotypiques, tout comme les cribles génétiques, ressemblent à une pêche à la ligne. C'est pourtant une approche puissante et unique pour identifier des acteurs clés et inédits d'une fonction biologique d'intérêt.
Les méthodes employées pour les criblages phénotypiques sont très variées: tests sur cellules vivantes, sur des organismes modèles (drosophile, poisson zèbre, vers C. elegans) ou sur des extraits complexes (extraits d'œufs de xénope). Si les microplaques restent le format de choix pour la réalisation des tests, il faut signaler des technologies en émergence, basées par exemple sur les progrès de la microfluidique, qui permettent de diminuer la quantité de réactifs et d'augmenter la cadence [7]. Tout en conservant une pertinence fonctionnelle, il faut miniaturiser ces tests, parfois complexes, afin de pouvoir les réaliser dans un format adapté à une utilisation robotisée. Quelque soit le format choisi, les tests doivent aussi présenter des qualités de robustesse et de sensibilité compatibles avec une analyse statistique des données (Figure 3) [8].

La nature et la complexité du test affectent la cadence (nombre de plaques criblées par jour) et seront donc des critères déterminants dans le choix de la taille des collections à cribler. Par exemple, l'acquisition automatique d'images en microplaques, couplée à une analyse d'image automatisée (high content analysis, HCA) permet des criblages riches en informations (high content screening, HCS) (Figure 4). Toutefois, la complexité et le temps nécessaire à l'analyse des images constituent un frein pour l'utilisation du HCS avec de larges collections de molécules. Le HCS est, ainsi, souvent réservé au crible de chimiothèques ciblées $[9,10]$. 


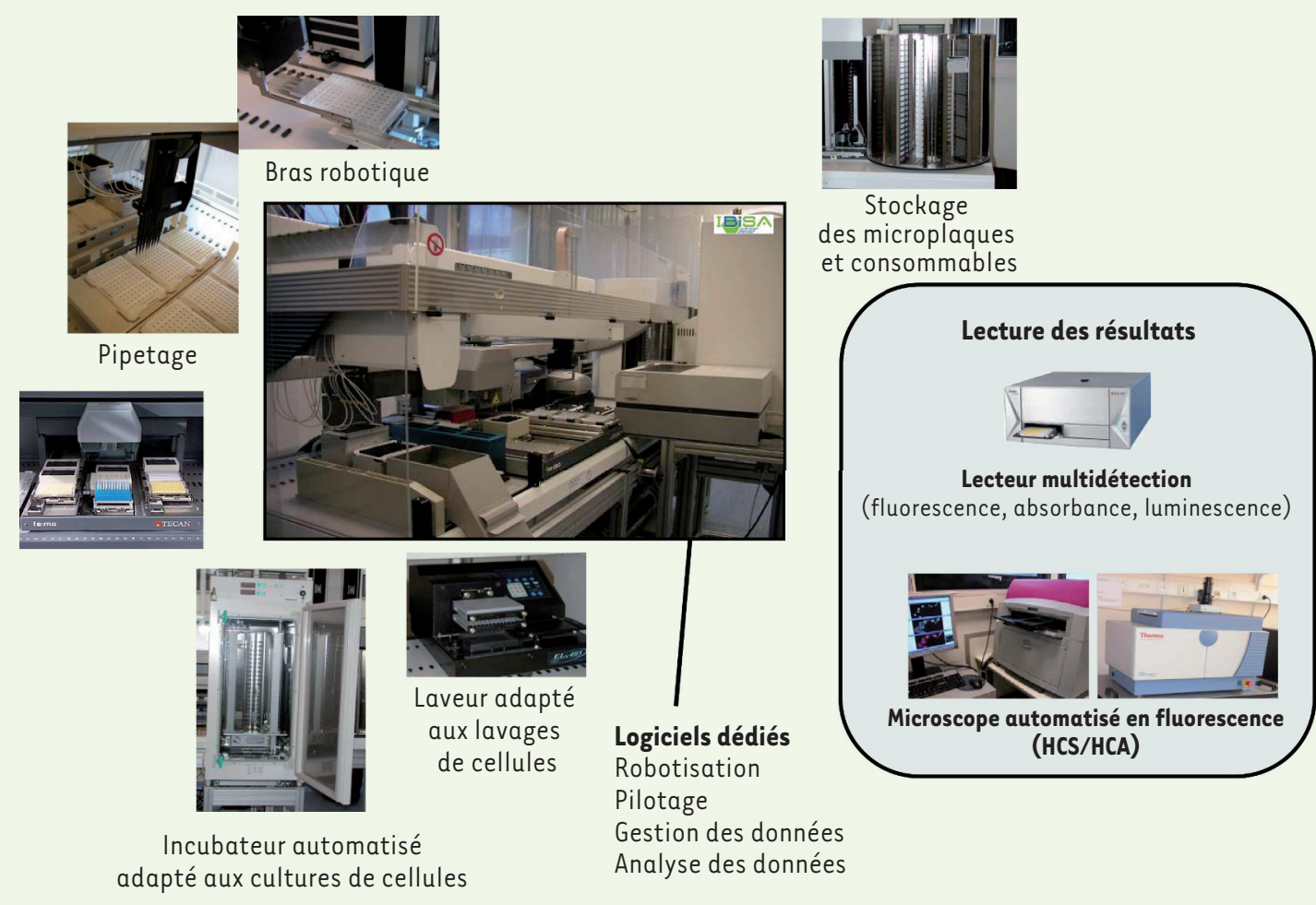

Figure 2. Le Centre de criblage pour des molécules bio-actives (CMBA) : un exemple d'une plate-forme de criblage automatisé. La plate-forme est constituée d'un robot central de pipetage et de manipulation. Elle intègre un ensemble d'appareils périphériques. Installée sous un flux laminaire, elle est conçue et équipée pour la réalisation de tests sur cellules. Les résultats sont obtenus grâce à un lecteur multidétection (signaux de fluorescence, absorbance, luminescence) directement connecté à la plateforme. Des microscopes automatisés à fluorescence sont également disponibles pour les acquisitions d'images de tests phénotypiques.

L'élaboration du test phénotypique est une étape longue et cruciale pour le résultat final. Plus le test rendra compte d'un phénotype précis, plus les molécules actives sélectionnées seront pertinentes, et plus l'analyse de leur mécanisme d'action sera facilitée. Ainsi, un simple test de toxicité est généralement insuffisant car de nombreux facteurs affectent la croissance et la mort cellulaire. Cependant, un test de toxicité réalisé parallèlement sur deux lignées cellulaires identiques ne différant que par un facteur (mutation d'un gène, exposition à une molécule, etc.) peut s'avérer pertinent [11].

L'emploi de plusieurs marqueurs dans un même test est un bon compromis entre quantité d'informations recueillie et cadence. Ainsi, nous avons développé un test multiparamétrique visant à repérer des molécules actives sur les microtubules cellulaires [12]. Ce test, associé à une stratégie de postcriblage adaptée, a permis la découverte de molécules ciblant directement la tubuline [13] ou des protéines régulatrices de la dynamique microtubulaire [5] (Figure 5).

\section{Chimiothèques}

La probabilité d'élucider de nouveaux mécanismes augmente, en théorie, avec l'emploi de chimiothèques dotées d'une diversité et d'une complexité structurales élevées (Tableau I). Le caractère essentiel de la diversité structurale est lié au fait que l'ensemble des composants cellulaires (protéines, ARN, etc.) constitue des cibles potentielles. De plus, ces molécules biologiques présentent des topologies complexes, résultant du repliement des chaînes polypeptidiques ou nucléotidiques. Cela génère des cavités aux formes complexes. Afin de présenter une affinité suffisante, les ligands de ces cavités doivent donc avoir des fonctions chimiques judicieusement positionnées pour une interaction optimale. Cela peut être obtenu par l'emploi de molécules dont la complexité structurale est élevée. L'obtention de telles molécules peut nécessiter le développement de nouvelles méthodes de synthèses (chimie radicalaire, couplage organométallique, photochimie) permettant d'accéder plus rapidement à des squelettes jusqu'alors difficilement accessibles. Certaines molécules d'origine naturelle présentent également une complexité structurale élevée (paclitaxel, morphine, etc.).

La complexité structurale est notamment favorable pour moduler des interactions protéine-protéine qui jouent 


\begin{tabular}{|c|c|c|c|c|c|}
\hline $\begin{array}{l}\text { Nom de } \\
\text { la collection } \\
\text { de composés }\end{array}$ & Fournisseur & $\begin{array}{c}\text { Statut } \\
\text { du } \\
\text { fournisseur }\end{array}$ & $\begin{array}{l}\text { Nombre de } \\
\text { composés }\end{array}$ & Caractéristiques & À noter \\
\hline $\begin{array}{l}\text { Chimiothèque } \\
\text { nationale }\end{array}$ & $\begin{array}{l}\text { Unité de gestion } \\
\text { de la chimio- } \\
\text { thèque natio- } \\
\text { nale, CNRS UPS } \\
3035\end{array}$ & Académique & 52894 & $\begin{array}{l}41225 \text { composés et } \\
14514 \text { extraits, issus de } \\
39 \text { équipes de chimie du } \\
\text { CNRS, en constante } \\
\text { augmentation }\end{array}$ & $\begin{array}{l}\text { Accès à l'expertise du laboratoire } \\
\text { de chimie à l'origine des molécules } \\
\text { d'intérêt, utile pour l'étape } \\
\text { d'optimisation }\end{array}$ \\
\hline $\begin{array}{l}\text { Chimiothèque } \\
\text { nationale } \\
\text { essentielle }\end{array}$ & $\begin{array}{l}\text { Unité de gestion } \\
\text { de la chimio- } \\
\text { thèque natio- } \\
\text { nale, CNRS UPS } \\
3035\end{array}$ & Académique & 640 & $\begin{array}{l}\text { Diversité et représentati- } \\
\text { vité des substances de la } \\
\text { chimiothèque nationale } \\
\text { (voir ci-dessous) }\end{array}$ & $\begin{array}{l}\text { Accès à un certain nombre } \\
\text { d'analogues pour les étapes de } \\
\text { SAR et d'optimisation }\end{array}$ \\
\hline DIVERSet ${ }^{T M}$ library & $\begin{array}{l}\text { ChemBridge cor- } \\
\text { poration }\end{array}$ & Commercial & 16480 & $\begin{array}{l}\text { Composés drug-like d'une } \\
\text { grande diversité pharma- } \\
\text { cophorique }\end{array}$ & $\begin{array}{l}\text { Propose } 900000 \text { composés et les } \\
\text { collections à façon EXPRESS-Pick } \\
\text { Collection stock et CORE library } \\
\text { stock, basées sur la diversité } \\
\text { structurale et la couverture de } \\
\text { l'espace chimique }\end{array}$ \\
\hline $\begin{array}{l}\text { Prestwick chemical } \\
\text { library }^{\circledR}\end{array}$ & $\begin{array}{l}\text { Prestwick } \\
\text { Chemical }\end{array}$ & Commercial & 1200 & $\begin{array}{l}\text { Composés approuvés par } \\
\text { des agences telles que la } \\
\text { FDA, à haute drugability }\end{array}$ & $\begin{array}{l}\text { Propose aussi une collection } \\
\text { de } 320 \text { produits naturels, une } \\
\text { collection de composés approuvés } \\
\text { par la FDA et bien tolérés chez } C \text {. } \\
\text { elegans, ainsi que des collections } \\
\text { de peptides et de fragments }\end{array}$ \\
\hline $\begin{array}{l}\text { Tocriscreen kinase } \\
\text { inhibitor toolbox }\end{array}$ & TocrisBioscience & Commercial & 80 & $\begin{array}{l}\text { Inhibiteurs connus de } \\
\text { kinases et de phospha- } \\
\text { tases }\end{array}$ & $\begin{array}{l}\text { Propose une collection de } \\
1120 \text { composés bio-actifs et la } \\
\text { possibilité de définir une collection } \\
\text { à façon }\end{array}$ \\
\hline $\begin{array}{l}\text { ICCB known } \\
\text { bioactives library }\end{array}$ & $\begin{array}{l}\text { Enzo Life } \\
\text { Sciences }\end{array}$ & Commercial & 480 & $\begin{array}{l}\text { Composés bio-actifs } \\
\text { développés en coopération } \\
\text { avec le Harvard institute of } \\
\text { chemistry and cell biology }\end{array}$ & $\begin{array}{l}\text { Propose aussi de nombreuses } \\
\text { petites collections ciblées de } \\
\text { molécules (enzymes, voies de } \\
\text { signalisation, récepteurs orphelins, } \\
\text { etc.) et de produits naturels }\end{array}$ \\
\hline LOPAC® 1280 & Sigma & Commercial & 1280 & $\begin{array}{l}\text { Composés pharmacologi- } \\
\text { quement actifs }(250 \mu \mathrm{l} \text { à } \\
10 \mathrm{mM}) \text {; cette collection } \\
\text { est aussi proposée au for- } \\
\text { mat } 25 \mu \mathrm{l} \text { à } 10 \mathrm{mM} \text { (LOPAC® } \\
1280 \text { - small scale) }\end{array}$ & $\begin{array}{l}\text { Sigma propose aussi plus de } \\
4000 \text { composés bio-actifs }\end{array}$ \\
\hline Chemical library & $\begin{array}{l}\text { Selleck } \\
\text { Chemicals }\end{array}$ & Commercial & 1463 & $\begin{array}{l}\text { Composés bio-actifs; } \\
\text { cette collection est pro- } \\
\text { posée au format } 100 \mu \mathrm{l} \text { et } \\
250 \mu \mathrm{l} \text { à } 10 \mathrm{mM}\end{array}$ & $\begin{array}{l}\text { Propose aussi plusieurs plus } \\
\text { petites collections ciblées de } \\
\text { composés connus (enzymes, GPCR, } \\
\text { épigénétique, cellules souches, } \\
\text { etc.) }\end{array}$ \\
\hline $\begin{array}{l}\text { Targeted diversity } \\
\text { library }\end{array}$ & ChemDiv & Commercial & 50000 & $\begin{array}{l}\text { Molécules drug-like, sélec- } \\
\text { tionnées sur une centaine } \\
\text { de sous-ensembles }\end{array}$ & $\begin{array}{l}\text { Propose de nombreuses collections } \\
\text { ciblées; dispose de } 1425000 \\
\text { composés drug-like }\end{array}$ \\
\hline $\begin{array}{l}\text { Pre-plated diver- } \\
\text { sity sets }\end{array}$ & Life chemicals & Commercial & $\begin{array}{l}5120 \\
10240 \\
15040 \\
20160\end{array}$ & $\begin{array}{l}\text { Molécules sélectionnées } \\
\text { sur la base de la diversité } \\
\text { structurale et } \\
\text { la drug ability }\end{array}$ & $\begin{array}{l}\text { Propose de nombreuses collections } \\
\text { ciblées }\end{array}$ \\
\hline
\end{tabular}

Tableau I. Liste non exhaustive de différentes chimiothèques et présentation de leurs caractéristiques principales. SAR : étude des relations structure-activité 


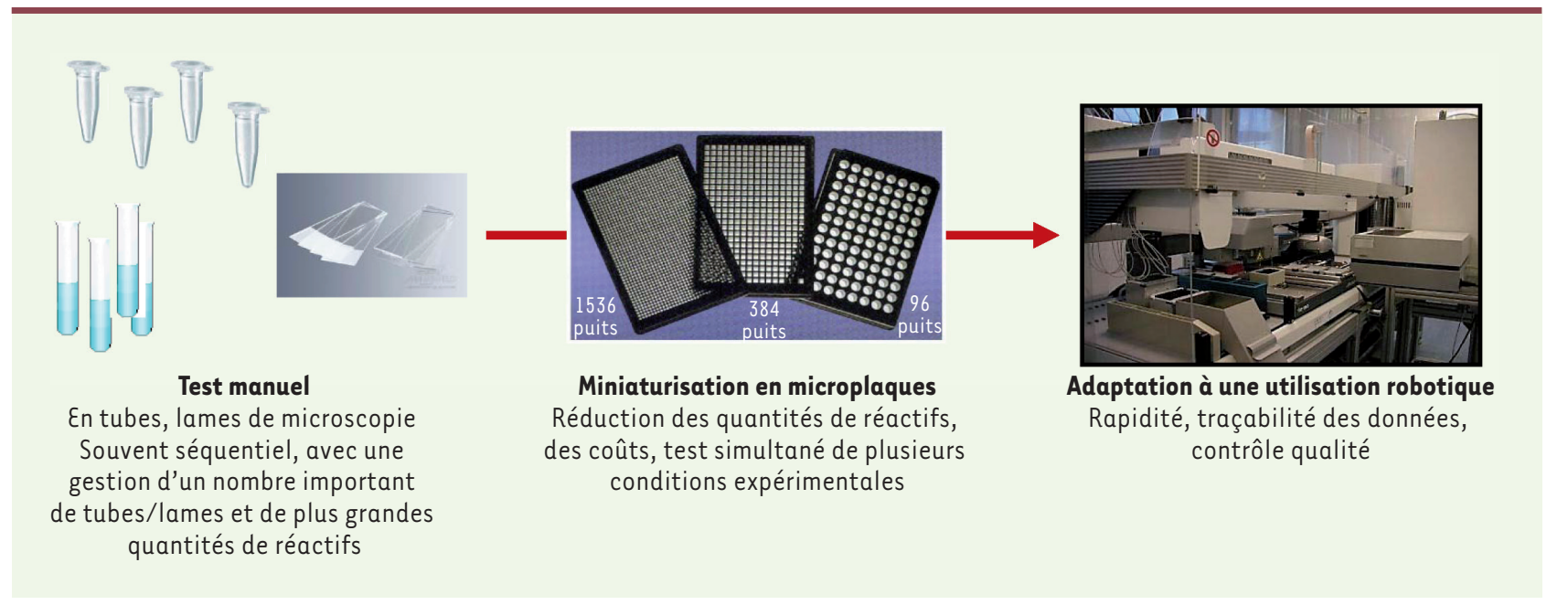

Figure 3. Schéma des étapes de l'adaptation d'un test manuel au format de microplaques (96, 384 ou 1536 puits) pour son exécution itérative par une plate-forme robotisée.

un rôle central dans de nombreux processus biologiques, et dont les surfaces d'interactions sont souvent larges et hydrophobes [14]. Cette diversité et cette complexité sont néanmoins contraintes par le vivant: les molécules doivent présenter une hydrophobicité adaptée pour franchir la membrane cellulaire. Enfin, il convient généralement d'éviter les molécules présentant une trop grande réactivité chimique (chlorure d'acyle, accepteurs de Michael, etc.), susceptibles d'interagir de manière non spécifique sur un large éventail de cibles cellulaires.

\section{Les collections de substances naturelles}

Dans le contexte du criblage sur cellule, les substances naturelles, biocompatibles et souvent diverses et complexes, semblent bien adaptées. Historiquement, le succès des substances naturelles provenant de diverses sources (extraits de venins, organismes marins, plantes tropicales, etc.) est indéniable [15]. Cependant, ces collections sont souvent constituées d'extraits complexes. II est ensuite nécessaire de réaliser une purification bio-guidée pour isoler le composé actif, consistant à fractionner les échantillons et à tester l'activité biologique de chaque extrait. Ce cycle est répété jusqu'à l'obtention d'un composé actif pur [16]. Bien qu'à l'origine de composés originaux, cette approche présente un certain nombre de biais. Tout d'abord, des composés peu abondants ou d'activité modérée, ne seront pas décelés. De plus, des composés cytotoxiques peuvent masquer l'activité d'autres composés. Enfin, des effets synergiques ou coopératifs entre différents composés peuvent produire une activité qui disparaîtra lors des fractionnements ultérieurs. La mise à disposition de collections de substances naturelles pures permet désormais de pallier ces problèmes.

\section{Les collections commerciales}

Ces collections peuvent être choisies selon différents critères (diversité, $\log \mathrm{P}^{1}$, etc.). Il existe également des chimiothèques ciblées

\footnotetext{
${ }^{1}$ Le logP est une mesure de la solubilité différentielle de composés chimiques dans deux solvants qui rend
} compte de la tendance d'une molécule à s'accumuler dans les membranes biologiques. (inhibiteurs de kinase, de protéases, pharmacophores privilégiés). Ces collections permettent de valider une approche et d'identifier des structures chimiques d'intérêt.

Plusieurs sociétés (Sigma, Prestwick chemicals, etc.) proposent également des collections constituées de molécules approuvées par la FDA (Food and drug administration). Ces collections peuvent permettre de valider un test, d'identifier des faux-positifs, de préciser la stratégie postcriblage et, parfois, de découvrir de nouvelles applications à des molécules pharmacologiques connues. De plus, les molécules trouvées actives à l'issue du criblage de ces collections peuvent orienter le choix de collections ultérieurement criblées, comme par exemple des collections de molécules présentant un pharmacophore donné. Ces considérations nous amènent à les recommander pour une validation préliminaire des criblages cellulaires.

\section{La chimiothèque nationale}

$\varepsilon n$ France, de nombreux laboratoires académiques de chimie ont regroupé et formaté en microplaques leurs productions (produits de synthèse, composés ou extraits naturels) afin de les rendre utilisables pour le criblage sur des tests biologiques. La chimiothèque nationale (CN) ainsi constituée est une fédération de chimiothèques². Cette démarche de valorisation du patrimoine scientifique vise à favoriser la découverte de molécules bioactives grâce à des recherches interdisciplinaires. La multiplicité des spécialités des chimies employées pour la synthèse des molécules constituant la CN (plus de 50000 molécules, en croissance permanente), la rend très

\footnotetext{
2 Pour informations, consulter http://chimiotheque-nationale.enscm.fr.
} 


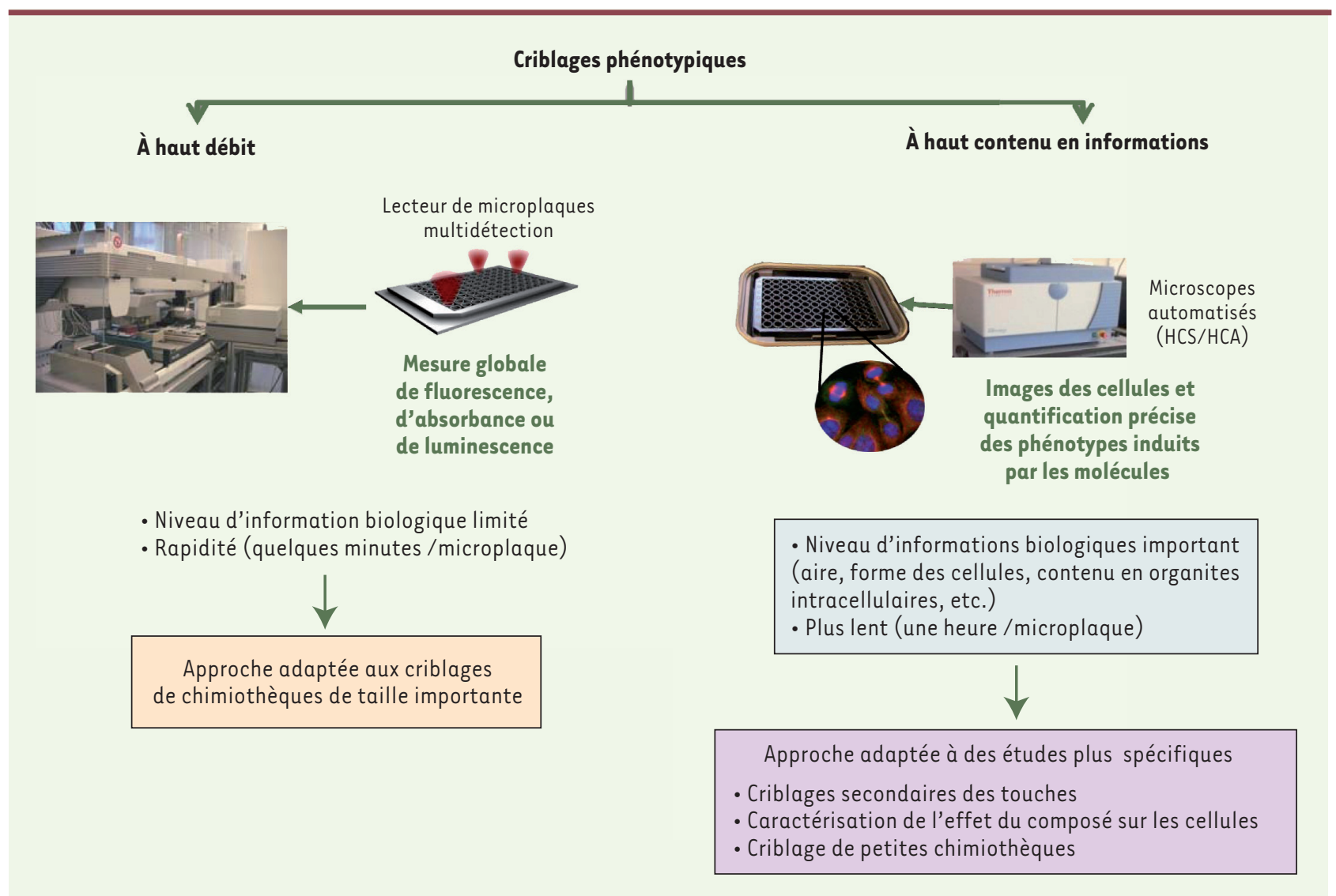

Figure 4. Intérêts et contraintes du high content screening (HCS). De par la richesse des données générées, le HCS a fait ses preuves pour la découverte de nouvelles molécules bio-actives et de nouvelles cibles biologiques. Cependant, la vitesse d'acquisition et d'analyse d'images ont tendance à diminuer le débit des criblages automatisés. Au CMBA, nous avons fait le choix d'utiliser la plate-forme robotique équipée d'un lecteur multidétection pour les criblages de grandes chimiothèques et d’utiliser le HCS pour des études plus spécifiques et plus ciblées.

diverse. Les efforts des chimistes sensibilisés aux criblages ont permis de proposer à la communauté des chimiothèques spécialisées. Par exemple, un ensemble de composés divers et fluorescents, peuvent ensuite servir de rapporteurs pour des expériences de compétition [17]. Cela a permis, notamment, la découverte de ligands fortement affins pour des récepteurs orphelins. D'autres chimiothèques, exploitant les avancées en chimie organométallique, sont enrichies en composés destinés à cibler les interactions protéine-protéine $[14,18]$. Outre son intérêt en termes de diversité et d'originalité, l'utilisation de la CN génère des partenariats fructueux entre chimistes et biologistes pour, sur le long terme, l'étude et la valorisation des molécules sélectionnées.

\section{Identification de la cible}

L'identification des cibles protéiques des molécules issues des criblages est un enjeu de la génétique chimique. Suite à la sélection d'une molécule, une série de tests complémentaires est nécessaire pour restreindre le champ d'investigations. Ainsi, après le criblage décrit plus haut [12], les molécules actives sur les microtubules ont été testées sur l'assemblage in vitro de la tubuline, afin de séparer celles qui agissent directement de celles qui agissent de manière indirecte sur la tubuline, ces dernières ayant ensuite été soumises à de nouveaux tests fonctionnels en vue d'identifier leur cible biologique.

Ces tests complémentaires appropriés sont cruciaux pour la réussite de ces recherches. En effet, il n'existe pas de méthode standard pour identifier une cible. Cela nécessite l'emploi de différentes techniques qui sont choisies selon la question scientifique posée, la structure de la molécule ainsi que le phénotype observé. La diversité des techniques mises en œuvre pour l'identification de la cible biologique d'une molécule chimique nécessite souvent des approches pluridisciplinaires. Elles se divisent en deux classes : (1) celle visant à détecter une interaction directe entre la cible et le composé d'intérêt ; et (2) celle visant à définir les acteurs cellulaires touchés par la molécule et responsables du phénotype d'intérêt (composants d'une voie de signalisation par exemple).

\section{Recherche de la cible par des études d'interaction} Les techniques de la première classe sont majoritairement biophysiques ou biochimique : ce sont les approches de chromatographie d'affinité, visant à purifier la cible 


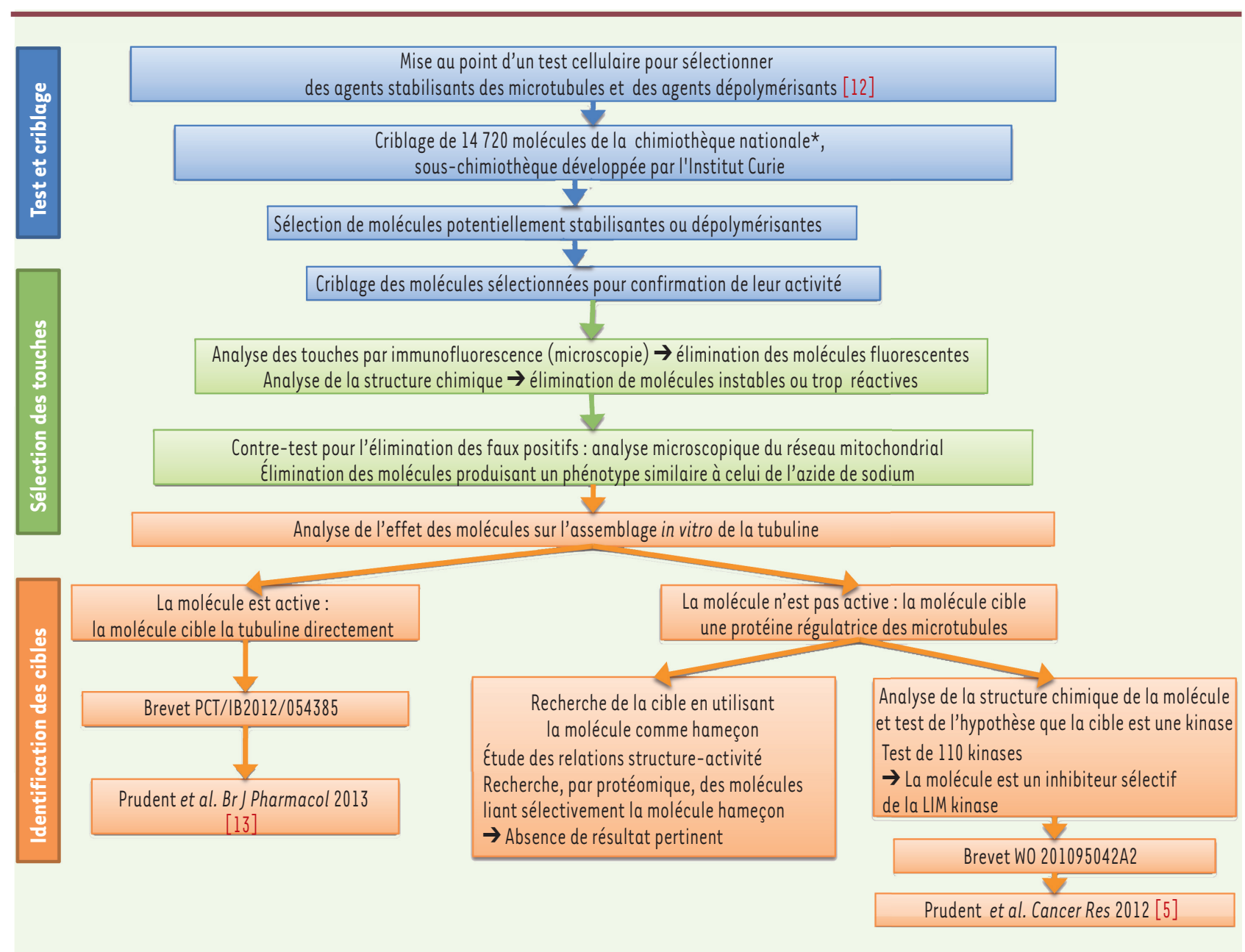

Figure 5. Les différentes étapes d'une approche de chémobiologie pour l'identification de régulateurs du cytosquelette. *http://chimiothequenationale.enscm.fr/

grâce à un ligand immobilisé sur un support ou grâce à un ligand marqué $[19,20]$. Différentes méthodologies (séquençage, outils immunologiques, analyse protéomique) permettent ensuite d'identifier les protéines liées à la molécule, constituant les cibles potentielles. Cette approche implique la synthèse d'analogues modifiés demeurants actifs. Toutefois, il est important, lors de ces analyses, de garder à l'esprit que la cible peut être un assemblage supramoléculaire. Ceci peut compliquer l'identification de la cible à partir d'extraits cellulaires. De plus, ce genre de purification ne permet pas toujours de faire la distinction entre une cible cellulaire abondante et de faible affinité, et une cible présentant une plus forte affinité, mais peu représentée.

D'autres méthodes reposent sur la stabilité différentielle des protéines en réponse à la fixation d'un ligand (méthode DARTS [drug affinity responsive target stability] [21]), la protéomique quantitative, ou encore I'interaction du composé marqué avec des puces à protéines [22]. Dans le cas où la structure moléculaire est caractéristique de molécules ciblant une classe de protéines (kinases, protéases, actine, tubuline, phosphatase, GPCR [G protein-coupled receptor], etc.), des tests in vitro sur ces cibles peuvent s'avérer très fructueux.
La connaissance qu'a le biologiste du système qu'il explore joue aussi un rôle central. C'est ainsi que Mayer et al. [3] ont identifié la cible du monastrol: la kinésine Eg5. En effet, l'addition de ce composé entraîne la formation d'un fuseau mitotique monopolaire (mono-astral), au lieu du fuseau bipolaire normal. Cette forme de fuseau avait déjà été observée lorsque le moteur $\varepsilon g 5$ est bloqué par un anticorps inhibiteur. Le test in vitro de l'effet du monastrol sur la kinésine $\varepsilon g 5$ a permis de démontrer que le monastrol était un inhibiteur sélectif de ce moteur.

\section{Recherche de la cible par des études d'identification d'acteurs phénotypiques}

Le deuxième type de stratégie semble, de prime abord, plus indirect et vise à identifier les acteurs les plus proches possibles de l'élément générateur du phénotype. Ces stratégies incluent les techniques suivantes:

- Complémentation/suppression biochimique dans laquelle un extrait cellulaire est fractionné et les 


\section{ChemBioScreen : un réseau unique réunissant les compétences françaises en criblage}

Depuis le début des années 2000 , de nombreuses équipes françaises se sont investies dans les approches de chémobiologie. Les criblages sont réalisés sur des plateaux techniques de laboratoire, ou bien, à l'image du CMBA, sur des plates-formes affichant une volonté d'ouverture et de collaboration avec un grand nombre de laboratoires (plates-formes IBiSA).

Ces travaux débouchent sur un nombre croissant de publications, démontrant, si besoin était, leur utilité tant d'un point de vue fondamental que médical. Ainsi, sur les 4 dernières années, une soixantaine de travaux issus de laboratoires français et reposant sur un criblage ont été publiés, pour certains dans des revues à très haut facteur d'impact [28-30]. Parallèlement, ces travaux ont fait l'objet d'une vingtaine de brevets. Le délai de plusieurs années entres les étapes initiales de criblage et la concrétisation des résultats sous forme de publication, traduit, d'une part, la nouveauté de la discipline, et, d'autre part, la complexité de la mise en œuvre de l'ensemble de la stratégie.

Conscients que ce type d'approche impose un savoir-faire unique - pour s'adapter aux contraintes de la miniaturisation du test, à l'analyse d'un grand nombre de données, à la gestion de chimiothèques, au développement de nouvelles méthodes de détection et d'analyse [10, 31, 32] et suivre une démarche qualité - ainsi qu'une ouverture à l'interdisciplinarité (interface chimie-biologie, pharmacochimie, statistiques, chemoinformatique, etc.), les acteurs du criblage ont, dès 2002, manifesté leur souhait de partager leur savoir-faire et d'aider à la formation des équipes souhaitant s'investir dans ces approches. Cette volonté s'est traduite par l'organisation d'écoles thématiques bisannuelles, qui rencontrent un vif succès, témoignant de la vitalité de la communauté scientifique s'intéressant aux approches de chémobiologie. Depuis 2 ans, grâce au soutien du CNRS, cette communauté s'est structurée sous forme d'un Groupement de Recherche (GDR), «ChemBioScreen ». Ce GDR regroupe actuellement 24 laboratoires (voir carte, Figure 6) et accueillera bientôt 2 nouvelles équipes (www.chembioscreen.fr).

En plus de son rôle d'animation, d'échanges de savoir faire, de formation autour des approches de chémobiologie, le GDR «ChemBioScreen » a pour objectif d'interagir étroitement avec d'autres structures comme le GDR Chemoinformatique, le GIS « Chimiothèque Nationale », le GDR «BioChiMar», la communauté des utilisateurs du HCS/HCA, etc.

Avec le recul dont nous disposons désormais, il apparaît qu'un facteur clé du succès des criblages phénotypiques, et plus généralement des criblages de petites molécules, est la mise en œuvre d'interactions fluides entre les acteurs des différentes disciplines, aboutissant à des résultats majeurs contribuant à l'accroissement des connaissances sur le vivant, à l'origine de publications de haut niveau, de brevets et, nous l'espérons, de progrès thérapeutiques. Ainsi, les approches intégrées reposant sur une réelle synergie entre les différents acteurs s'avèrent les plus fructueuses.

fractions sont soumises à un test d'activité en présence du composé d'intérêt. Les fractions modifiant la réponse aux tests comportent alors un élément impliqué dans l'effet du composé [23].

- Combinaison de molécules : des molécules de référence sont appliquées conjointement avec le composé d'intérêt. L'étude des profils de réponse permet de situer l'élément sensible au composé d'intérêt (dans une voie de signalisation par exemple) par rapport aux cibles connues des molécules de référence [24]. Cette approche, similaire aux études d'épistasie en génétique, a permis de trouver des cibles de composés naturels.

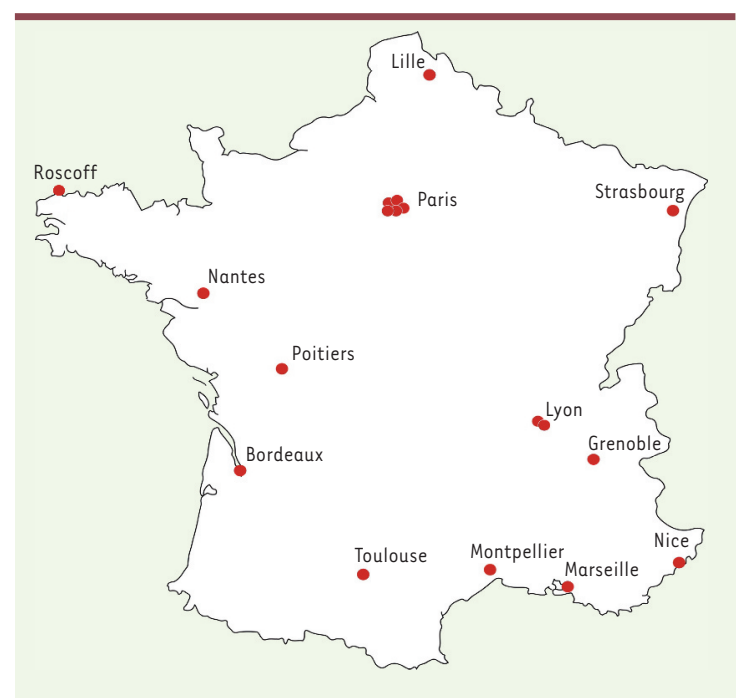

Figure 6. Localisation des différentes équipes formant le GDR ChemBioScreen.

- Comparaison du phénotype induit par la molécule avec les phénotypes de mutants de perte partielle ou totale de fonction (cellules traitées par des si/shARN ou organismes modèles) ou induits par des molécules chimiques dont on connaît la cible (comparaison des signatures pharmacologiques). Ces méthodes réclament l'accès à des bases de données exhaustives [25].

- Comparaison de la sensibilité de banque de mutants de S. cerevisiae aux composés [26]. La cible de composés anticancéreux, impliquée dans le métabolisme des sphingolipides, a ainsi été identifiée.

- Analyse du profil différentiel d'expression des protéines (par exemple par une approche par spectrométrie de masse type SILAC [stable isotope labeling by aminoacids in cell culture]) ou transcriptomique.

L'avantage de ces stratégies est de toujours considérer le phénotype étudié comme critère de sélection, ce qui limite les risques d'orienter les recherches de la cible sur de fausses pistes. Leur inconvénient est la nécessité de tester ensuite chacune des cibles potentielles isolées, individuellement.

\section{Limites de ces techniques de recherche de la cible}

Quelle que soit la stratégie développée, il convient de rester prudent devant les résultats obtenus. En effet, la spécificité d'une molécule vis-à-vis d'une protéine cible reste difficile à établir. Par exemple, une molécule complexe et de taille importante, comme le Taxol ${ }^{\circledR}$, dont le mécanisme d'action paraissait indiscutable (fixation avec une très forte affinité sur la tubuline), a d'autres cibles cellulaires, comme la protéine antiapoptotique $\mathrm{Bcl}-2$. Ces interactions, souvent qualifiées de secondaires, pourraient aussi contribuer à l'effet 
antiprolifératif du Taxol ${ }^{\circledR}$. Elles ne sont donc pas nécessairement problématiques, peuvent même s'avérer être un atout supplémentaire en thérapie, et méritent d'être décrites [27]. Pour d'autres molécules, ce type d'interactions secondaires peut être à l'origine d'effets phénotypiques inexpliqués, éventuellement rédhibitoire pour leur utilisation en thérapie ou en recherche.

\section{Conclusion et perspectives}

Développées il y a plus de 10 ans et initialement considérées comme risquées, les approches basées sur le criblage phénotypique bénéficient de nombreux développements biologiques, chimiques, statistiques, technologiques, mais également humains. En effet, depuis deux ans, grâce au soutien du CNRS, une communauté de scientifiques s'est structurée sous la forme du Groupement de recherche (GDR) ChemBioScreen (Figure 6). Ce GDR regroupe actuellement 24 laboratoires (Figure 6), et accueillera bientôt deux nouvelles équipes.

Les approches basées sur le criblage phénotypique jouent également un rôle central dans la découverte de nouveaux mécanismes biologiques. Elles ont aussi permis l'identification de nouvelles cibles thérapeutiques, accessibles aux petites molécules. Si, à l'issue du criblage, ces dernières sont encore loin de représenter un futur médicament, elles présentent néanmoins, de part leur méthode de sélection, des caractéristiques de biodisponibilité utiles pour l'analyse approfondie de leur mécanisme d'action et de leur efficacité thérapeutique. $\diamond$

\section{SUMMARY}

Phenotypic screens or one stone to kill two birds:

discover the target and its pharmacological regulator

Phenotypic screens, in which chemical libraries are assayed on cells with the aim to isolate compounds that interfere with a given cell function, are a risky but powerful strategy to discover, in the same approach, new therapeutic targets and the compounds able to regulate them. With a strong experience of nearly 10 years in the field, we present the advantages of such an approach, the possible troubles and technical solutions. We also present in this paper a french network which has been recently built and that gather all the competencies needed for screening approaches. $\diamond$

\section{LIENS D'INTÉRÊT}

Les auteurs déclarent n'avoir aucun lien d'intérêt concernant les données publiées dans cet article.

\section{RÉFÉRENCES}

1. Peterson JR, Mitchison TJ. Small molecules, big impact : a history of chemical inhibitors and the cytoskeleton. Chem Biol $2002 ; 9$ : 1275-85.

2. Eggert US. The why and how of phenotypic small-molecule screens. Nat Chem Biol $2013 ; 9: 206-9$.

3. Mayer TU, Kapoor TM, Haggarty SJ, et al. Small molecule inhibitor of mitotic spindle bipolarity identified in a phenotype-based screen. Science $1999 ; 286: 971-4$.

4. Lefurgy S, Cornish V. Finding Cinderella after the ball : a three-hybrid approach to drug target identification. Chem Biol $2004 ; 11$ : 151-3.

5. Prudent R, Vassal-Stermann $\varepsilon$, Nguyen CH, et al. Pharmacological inhibition of LIM kinase stabilizes microtubules and inhibits neoplastic growth. Cancer Res $2012 ; 72: 4429-39$.

6. Swinney DC, Anthony J. How were new medicines discovered? Nat Rev Drug Discov $2011 ; 10$ : 507-19.
7. Miller 0J, દl Harrak A, Mangeat T, et al. High-resolution dose-response screening using droplet-based microfluidics. Proc Natl Acad Sci USA 2012 ; $109: 378-83$.

8. Malo N, Hanley JA, Cerquozzi S, et al. Statistical practice in high-throughput screening data analysis. Nat Biotechnol $2006 ; 24$ : 167-75.

9. Soleilhac $\varepsilon$, Nadon R, Lafanechere L. High-content screening for the discovery of pharmacological compounds : advantages, challenges and potential benefits of recent technological developments. Expert Opin Drug Discov $2010 ; 5: 135-44$.

10. Lo $\varepsilon$, Soleilhac $\varepsilon$, Martinez A, et al. Intensity quantile estimation and mapping-a novel algorithm for the correction of image non-uniformity bias in HCS data. Bioinformatics $2012 ; 28: 2632-9$.

11. Raj L, Ide T, Gurkar AU, et al. Selective killing of cancer cells by a small molecule targeting the stress response to ROS. Nature 2011 ; 475 : 231-4.

12. Vassal $\varepsilon$, Barette $C$, Fonrose $X$, et al. Miniaturization and validation of a sensitive multiparametric cell-based assay for the concomitant detection of microtubule-destabilizing and microtubule-stabilizing agents. J Biomol Screen 2006 ; 11 : 377-89.

13. Prudent $\mathrm{R}$, Vassal-Stermann $\varepsilon$, Nguyen $\mathrm{CH}$, et al. Azaindole derivatives are inhibitors of microtubule dynamics, with anti-cancer and anti-angiogenic activities. BrJ Pharmacol $2013 ; 168: 673-85$.

14. Morelli X, Bourgeas R, Roche P. Chemical and structural lessons from recent successes in protein-protein interaction inhibition (2P21). Curr Opin Chem Biol $2011 ; 15: 475-81$.

15. Harvey AL. Natural products as a screening resource. Curr Opin Chem Biol $2007 ; 11: 480-4$.

16. Fonrose $X$, Ausseil F, Soleilhac $\varepsilon$, et al. Parthenolide inhibits tubulin carboxypeptidase activity. Cancer Res 2007 ; 67 : 3371-8.

17. Loison S, Cottet M, Orcel H, et al. Selective fluorescent nonpeptidic antagonists for vasopressin $V_{2}$ GPCR : application to ligand screening and oligomerization assays. J Med Chem 2012 ; 55 : 8588-602.

18. Laudet $B$, Prudent $R$, Filhol 0 , Cochet $C$. Des agents thérapeutiques ciblant des interactions protéine-protéine. Med Sci (Paris) 2007 ; 23 : 273-8.

19. Guiffant D, Tribouillard D, Gug F, et al. Identification of intracellular targets of small molecular weight chemical compounds using affinity chromatography. Biotechnol J $2007 ; 2: 68-75$.

20. Titov D V, Liu JO. Identification and validation of protein targets of bioactive small molecules. Bioorg Med Chem $2012 ; 20$ : 1902-9.

21. Lomenick B, Hao R, Jonai N, et al. Target identification using drug affinity responsive target stability (DARTS). Proc Natl Acad Sci USA 2009; 106 : 21984-9.

22. Terstappen GC, Schlüpen C, Raggiaschi R, Gaviraghi G. Target deconvolution strategies in drug discovery. Nat Rev Drug Discov 2007 ; 6 : 891-903.

23. Peterson JR, Lebensohn AM, Pelish HE, Kirschner MW. Biochemical suppression of small-molecule inhibitors : a strategy to identify inhibitor targets and signaling pathway components. Chem Biol 2006 ; 13 : 443-52.

24. Lehár J, Zimmermann GR, Krueger AS, et al. Chemical combination effects predict connectivity in biological systems. Mol Syst Biol $2007 ; 3: 80$.

25. Neumann B, Walter T, Hériché JK, et al. Phenotypic profiling of the human genome by time-lapse microscopy reveals cell division genes. Nature 2010 ; $464: 721-7$.

26. Baetz K, McHardy L, Gable K, et al. Yeast genome-wide drug-induced haploinsufficiency screen to determine drug mode of action. Proc Natl Acad Sci USA $2004 ; 101:$ 4525-30.

27. Ferlini C, Cicchillitti L, Raspaglio G, et al. Paclitaxel directly binds to $\mathrm{Bcl}-2$ and functionally mimics activity of Nur77. Cancer Res $2009 ; 69: 6906-14$.

28. Stechmann B, Bai SK, Gobbo $\varepsilon$, et al. Inhibition of retrograde transport protects mice from lethal ricin challenge. Cell $2010 ; 141: 231-42$.

29. Botté Cy, Deligny M, Roccia A, et al. Chemical inhibitors of monogalactosyldiacylglycerol synthases in Arabidopsis thaliana. Nat Chem Biol $2011 ; 7$ : 834-42.

30. Willand N, Dirié B, Carette X, et al. Synthetic EthR inhibitors boost antituberculous activity of ethionamide. Nat Med $2009 ; 15: 537-44$.

31. Maurel D, Comps-Agrar L, Brock C, et al. Cell-surface protein-protein interaction analysis with time-resolved FRET and snap-tag technologies: application to GPCR oligomerization. Nat Methods 2008 ; 5 : 561-7.

32. Pauwels $\varepsilon$, Surdez D, Stoll G, et al. A probabilistic model for cell population phenotyping using HCS data. PloS One2012; 7 : e42715.

\section{TIRÉS À PART}

L. Lafanechère 